\title{
Attitudes Towards Accreditation and Quality Improvement Activities Among Hospital Employees in Iran: A Quantitative Study
}

This article was published in the following Dove Press journal: Journal of Multidisciplinary Healthcare

\author{
Edris Kakemam ' \\ Mohammad Reza Rajabi (iD ${ }^{2}$ \\ Pouran Raeissi ${ }^{3}$ \\ Lars Holger Ehlers ${ }^{4}$ \\ 'Department of Health Management and \\ Economics, School of Public Health, \\ Tehran University of Medical Sciences, \\ Tehran, Iran; ${ }^{2}$ Department of Cardiology, \\ Faculty of Medicine, Shahed University, \\ Tehran, Iran; ${ }^{3}$ Department of Health \\ Services Management, School of Health \\ Management and Information Sciences, \\ Iran University of Medical Sciences, \\ Tehran, Iran; ${ }^{4}$ Danish Center for \\ Healthcare Improvements, Institute of \\ Business and Management, Faculty of \\ Social Sciences, Aalborg University, \\ Aalborg, Denmark
}

Correspondence: Edris Kakemam Department of Health Management and Economics, School of Public Health, Tehran University of Medical Sciences, Tehran, Iran

Email edriskakemam@gmail.com
Purpose: The hospital accreditation system in Iran is relatively young, having been introduced in 2012. Therefore, there is a real need for research on the status and impact of hospital accreditation in Iran. The purpose of this study was to evaluate and compare attitudes towards accreditation and quality improvement activities among hospital employees, specifically the attitudes towards the impact of accreditation on the quality of healthcare and its benefits in Iran.

Materials and Methods: A cross-sectional survey was carried out at 23 teaching hospitals in three metropolises in Iran, all of which successfully passed national accreditation surveys. Some 1213 hospital managers, administrative staff, nurses, and para-clinical staff participated in the survey. The main outcome measures were quality results, and the activities related to quality improvement include senior managers' commitment and support, strategic quality planning, education and training, rewards and recognition, quality management, use of data, the involvement of professionals in accreditation, and accreditation benefits. The questionnaire was applied using a 5-point Likert scale ranging from 1 "strongly disagree" to 5 "strongly agree". One-way analysis of variance (ANOVA) was used to compare mean values between respondent groups.

Results: Among nurses and managers, there was low support for accreditation and even less among para-clinical staff who fail to see accreditation having a positive impact on healthcare quality. Also, nurses' attitudes toward the accreditation benefits were more positive compared with the two other groups. Staff stated that the main reasons for low support were a lack of education and training to act upon the accreditation survey results and a lack of management visibility and support for quality improvement.

Conclusion: Improving quality through means of hospital accreditation is a complex process with high demands for management and employees. Questionnaires on employees' attitudes and perceptions of the impact of accreditation and quality improvement-related activities in the hospitals can provide valuable information on the current problems of a hospital accreditation program.

Keywords: accreditation, quality improvement, employees, surveys, Iran

\section{Background}

Despite the wide implementation of accreditation standards in the past 40 years and increased possibility of providing high-quality, safe healthcare across the world, ${ }^{1,2}$ we are unsure of the actual effect of accreditation programs on the improvement of clinical care, as well as health services and systems. ${ }^{3,4}$ Research into the effectiveness of quality measures have provided inconsistent results and there is a lack of evidence 
about the main reasons for these inconsistencies. ${ }^{5-12}$ A recent example is a US study of 4000 hospitals showing a lack of meaningful association between clinical and patient-experienced outcomes and the type of accreditation system. $^{13}$

Questionnaires on attitudes towards accreditation have been suggested as a means for studying the effectiveness of accreditation - partly because a large number of people working in healthcare can be asked directly about their opinion towards the effectiveness of accreditation - and partly because questions can be asked about the prerequisites for effectiveness. ${ }^{6}$ The latter includes all the organizational and management arrangements that needs to be in place in order to facilitate continuous quality improvement through accreditation systems.

The attitudes of employees towards hospital accreditation were examined in several studies. ${ }^{5-8,12,14}$ In a nationwide survey conducted in Denmark, overall attitudes toward accreditation were positive, but physicians were more skeptical. ${ }^{6}$ Bahadori et al noted that from nurse perspectives, accreditation had a positive impact on the Iranian military hospitals' quality results, ${ }^{5}$ while other studies have indicated that accreditation had no impact on the quality of care. ${ }^{10,15}$

The Iranian health system is organized across three levels: national, provincial (regional), and district. This system is basically a public cooperative system that many organizations such as MOHME, Social Security Organization, private sector, charities delivered healthcare. The three main sources of funding in the health system are the public budget, social health insurance, and out-ofpocket payment. The healthcare is provided by 924 hospitals among which 570 (70\% of total hospital beds) are affiliated to the the Ministry of Health and Medical Education (MOHME). ${ }^{16,17}$

Iranian accreditation was established in 2010 by the MOHME. It was implemented in two phases using 8104 criteria. ${ }^{18}$ During the first phase in 2011, the first draft standards were finalized and piloted in eight hospitals by the Office of Monitoring and Accreditation of Healthcare Institutions (OMAHI). ${ }^{15}$ After the pilot phase, the standards were modified using feedback. Eventually, the number of criteria was reduced to 2157. The type of standards was more structural and process-based, and outcome standards were very limited. ${ }^{18}$ Generally, tertiary and secondary healthcare services are provided in private and public hospitals, and all hospitals must obtain an operation license meeting specific procedural and structural standards. Therefore, participation in the accreditation process is mandatory for all hospitals. They then have to renew this license every year. Furthermore, an accreditation process must be carried out in hospitals every year to confirm their competencies in healthcare provision. ${ }^{18-20}$

Evaluation, guidance, coordination, financial support, and determining policies regarding healthcare services are among the responsibilities of the MOHME at a national level. ${ }^{21}$ However, provincially, the duties are delegated to the medical universities of and carried out by the OMAHI. Hospitals receive fee-for-service reimbursement, and their tariff is determined according to their level of accreditation. In other words, the higher the score of hospital, the greater amount of tariffs determined for patients.

While Iran has aimed to tackle the OMAHI accreditation so that the quality of its hospitals is improved, there is a lack of evidence for accepting accreditation as the most efficient application of resources to enhance quality results and procedures. This is mainly due to the primary stage of research associated with a legality problem for managers and hospital policymakers.

To improve quality via hospital accreditation is a complex process that puts a high demand on management and all groups of personnel. If some aspects of the complex mechanisms for continuous quality improvement are not implemented, or do not receive enough attention from management, the effectiveness of the whole system may be jeopardized. For both decision-makers and researchers, it is important to know which aspects of the accreditation system are more likely to fail and where improvements could be made.

The primary purpose of the study is to evaluate and compare the attitudes of hospital employees in Iran towards the impact of accreditation and its potential benefits. Specifically, it analyses and discusses attitudes towards the impact of accreditation on quality, as well as the importance of organizational and management activities related to quality improvement at hospitals. We were looking for answers to the following three questions:

1. What is the impact of accreditation on the quality of healthcare according to hospital employees?

2. What is the attitude of employees towards accreditation benefits?

3. How do employees assess the current qualityrelated activities? 


\section{Methods}

\section{Study Design}

A cross-sectional survey design was adopted.

\section{Settings and Participants}

The settings for this study were 23 teaching hospitals that are accredited by the MOHME in Iran, selected in three metropolises: Tehran, Tabriz, and Ahvaz. These hospitals have more than 250 beds in emergency, neonatal, pediatric, surgery, and internal medicine, dialysis wards, intensive care unit, and critical care unit to provide specialised care to patients with, among others, cardiac, endocrine, pulmonary, gastrointestinal, neurological, and psychological disorders. All the hospitals are affiliated to the university health sector and are the principal teaching hospitals with the largest workforces.

The source population of the study included working employees in various hospital wards who were familiar with the hospital before and after the accreditation. Therefore, only those employees who started working in the hospital before it was accredited and who have been working in the hospital for at least 3 years were chosen. The sample was selected from the hospitals by simple random sampling. We enrolled all hospital managers, administrative staff, nurses, and para-clinical staff. Physicians were not included in the survey because we anticipated a very low degree of participation. Pomey et al noted that French physicians believed accreditation was an administrative activity and only nursing managers and other employees should cooperate in its implementation. ${ }^{22}$ The data were collected between August 2016 and September 2017.

\section{The Questionnaire}

A self-administered questionnaire, developed by El-Jardali et $\mathrm{al}^{8}$ based on the questions from two studies, Shortell et $\mathrm{al}^{23}$ and Pomey et al, was used in this study. ${ }^{22}$ In order to reflect the Iranian healthcare setting, only minor changes were applied in the questionnaire. This questionnaire is a valid and comprehensive tool for the current model of Iranian accreditation which was developed and based on models in the US, France, Lebanon, and Egypt.

The survey was organised in three parts: demographic variables, quality results, quality improvement activities and benefits of accreditation. The following five sociodemographic variables were used: age, gender, work experience in current hospital, educational status, and staff position.
The quality results contained five items about improvements in the quality of customer satisfaction, services provided by the administration, care provided to patients, services provided by clinical support departments, and maintaining high-quality health services.

The activities related to quality improvement were senior managers' commitment and support (nine items about senior managers' commitment, support and participation in accreditation and quality improvement), strategic quality planning activities (seven items on hospital quality improvement planning process), quality management activities (six items, including questions about service quality assessments and policies), rewards and recognition activities (three items), use of data (seven items on using information for quality improvement), education and training activities (three items), involvement of employees in accreditation (six items about staff participation).

Benefits of accreditation included nine items about the improvement of patient care, the motivation of staff, teamwork and collaboration, development of values, better use internal resources, better respond to the populations' needs, better respond to partners, and development of collaboration with partners in the health care system. Items were scored on a 5-point Likert scale ranging from 1 "strongly disagree" to 5 "strongly agree".

The questionnaire was to be more relevant to Iran in two steps. Firstly, the original tool was translated into Farsi by the authors. Secondly, the Farsi version was translated back into English by an independent translator who had never seen the original version before. The original questionnaire and back-translation were compared. After the questionnaires were checked and found to have the same meaning, the Farsi translation was accepted as valid. Then, some items were edited, added or removed, based on our accreditation program.

Next, a value of item-level content validity index (I-CVI) was assessed by 10 experts, who were asked 1) to give suggestions on the relevancy of each item to the definition, 2) to evaluate clarity and conciseness of the wording. The experts were recruited from the area of interest of this study, including four experts in quality improvement and accreditation, two lecturers with expertise in accreditation, and four hospital managers. The evaluation followed the process suggested by Polit et al in having experts rate each item on a 4-point Likert scale (not relevant, somewhat relevant, quite relevant, and very relevant) based on item clarity and conciseness. The ratings were used to calculate an item-level content validity 
index (I-CVI) and to determine if items should be revised or deleted. A criterion of 0.80 of I-CVI among the experts was selected for inclusion in the list of items. ${ }^{24}$ During this step, the content validity of the items with a score of $0.84 \%$ was approved for the I-CVI indices.

Finally, a pilot test of the preliminary instrument was conducted on staff who had similar characteristics of the sample. Some 42 samples were selected to complete the pilot survey. Cronbach's alpha coefficient was examined to determine the internal consistency of the scale which indicates how well the items fit together conceptually, ${ }^{25}$ with the acceptable value of $\geq 0.70 .{ }^{26}$ Cronbach's alpha coefficient ranged from 0.73 to 0.92 . Scales demonstrated good internal consistency in this study: $\alpha=0.76$ for quality results, $\alpha=0.91$ for senior managers' commitment and support, $\alpha=0.83$ for strategic quality planning, $\alpha=0.82$ for quality management, $\alpha=0.85$ for use of data, $\alpha=0.73$ for rewards and recognition, $\alpha=0.79$ education and training, $\alpha=0.81$ for involvement of employees in accreditation and $\alpha=0.92$ for Benefits of accreditation. Cutoff scores for all dimensions of accreditation were used. Scores of 2 and lower, 3 and lower, above 3 to 4 and 4 to 5 were allocated to the strong disagreement, disagreement, agreement, and strong agreement, respectively.

\section{Data Collection}

The study protocol was approved by the Ethics Committee of the Iran University of Medical Sciences. The participants taking part in the study were voluntary and all provided written informed consent. Also, we explained that the information would remain strictly confidential. The participating employees were asked to complete the questionnaire within 7 days either at home or in their workplace.

\section{Data Analysis}

Data were entered and analyzed using the statistical software IBM-SPSS (Statistical Package for Social Sciences), version 22.0 (Chicago, IL, USA). To describe the demographic characteristics of the respondents and their views on the survey tool items, various descriptive statistics such as mean, standard deviation, percentage, and frequency were performed. One-way analysis of variance (ANOVA) was used to compare mean values between respondent groups. Bonferroni correction for multiple comparisons was used post hoc in the analysis of variance. All the analysis was conducted with the level of significance set at $\mathrm{p}<0.05$.

\section{Results}

\section{Employees' Characteristics}

A total of 1670 printed questionnaires were distributed personally to hospital employees. Some 1213 were returned; a response rate of $72.6 \%$ (66-84\% across hospitals). The characteristics of the study sample are summarised in Table 1. The participants were female dominant (75.4\%). Most of the employees were aged between 30 and 39 (46.3\%), the mean age of participants was $33.1(\mathrm{SD}=7.2)$ years. Most respondents had graduated with undergraduate degrees $(80.2 \%)$ at the time of the study. In terms of work experience, most participants (69.4\%) had less than 10 years' experience. The average experience was $7.9(\mathrm{SD}=5.9)$ years.

\section{Impact of Accreditation on Quality results}

Table 2 shows the attitude of three groups of hospital personnel toward the impact of accreditation on quality results. In general, there is only low support from all groups, especially from para-clinical staff who fail to see the positive impact of accreditation.

Table I Demographic Characteristics of the Participants ( $N=1213$ )

\begin{tabular}{|l|l|l|}
\hline Characteristics & Frequency & Percentage \\
\hline Gender & & \\
Male & 298 & 24.6 \\
Female & 915 & 75.4 \\
\hline Age & & \\
$<30$ & 524 & 43.2 \\
$30-39$ & 562 & 46.3 \\
$\geq 40$ & 127 & 10.5 \\
\hline Educational status & & \\
Associate degree & 124 & 10.2 \\
Undergraduate & 973 & 80.2 \\
Master's or PhD degree & 116 & 9.6 \\
\hline Work experience in current & & \\
hospital (year) & & \\
$<$ 10 & 842 & 69.4 \\
I0-20 & 306 & 25.2 \\
$>20$ & 65 & 5.4 \\
\hline Staff position & & \\
Managers/ Staff & 216 & 17.8 \\
Clinical nurse & 757 & 62.4 \\
Para-clinical staff (radiologists and & 240 & 19.8 \\
laboratory experts) & & \\
\hline
\end{tabular}


Table 2 Hospital Employees' Attitudes Towards the Impact of Accreditation on Quality Results

\begin{tabular}{|c|c|c|c|c|}
\hline \multirow[t]{2}{*}{ After Accreditation, Which of the Following Has Been Improved? } & $\begin{array}{l}\text { Managers/ } \\
\text { Staff }\end{array}$ & Nurses & $\begin{array}{l}\text { Para- } \\
\text { Clinical } \\
\text { Staff }\end{array}$ & \multirow[t]{2}{*}{ P-value } \\
\hline & Mean (SD) & $\begin{array}{l}\text { Mean } \\
(\mathrm{SD})\end{array}$ & Mean (SD) & \\
\hline Customer satisfaction & $3.16(0.96)$ & $3.37(0.86)$ & $3.15(1.02)$ & $<0.001$ \\
\hline The quality of services provided by the administration (finance, human resources, etc.) & $3.09(0.92)$ & $3.12(0.94)$ & $2.89(1.01)$ & 0.003 \\
\hline $\begin{array}{l}\text { The quality of care provided to patients (e.g. medical, surgical, obstetric and paediatric } \\
\text { patients) }\end{array}$ & $3.32(0.87)$ & $3.33(0.91)$ & $3.13(0.96)$ & 0.011 \\
\hline $\begin{array}{l}\text { The quality of services provided by clinical support departments such as laboratory, } \\
\text { pharmacy and radiology }\end{array}$ & $3.10(1.19)$ & $3.36(1.01)$ & $3.29(0.90)$ & 0.004 \\
\hline Maintaining a high quality health service despite financial constraints & $3.15(0.97)$ & $3.18(0.95)$ & $2.97(0.10)$ & 0.008 \\
\hline Average of the above 5 questions & $3.22(0.77)$ & $3.36(0.75)$ & $3.05(0.95)$ & $<0.001$ \\
\hline
\end{tabular}

The mean score of quality results was significantly higher for nurses, compared to the other groups $(\mathrm{p}<0.001)$. In addition, the attitude of the nurses was significantly more positive in all areas, compared to the other groups $(p<0.05)$. According to the results, the mean score of perception of service improvement after implementation of accreditation in all areas was within the range of 2.89-3.37 for all three groups.

Table 3 shows the perceived benefits of accreditation among three groups of hospital personnel. The mean scores of items were significantly higher for nurses, compared to the other groups $(\mathrm{p}<0.001)$.

\section{Quality Improvement-Related Activities}

Table 4 and Appendix 1 show the activities related to quality improvement in Iranian hospitals. Employees point at problems, especially issues with a lack of management visibility and education and training. All groups responded that they do not have good enough education and training to act upon the results of the accreditation surveys and management are seen as invisible with respect to quality improvement.
Again, the nursing staff had a more positive attitude towards all quality enhancement activities. Compared to the other two groups, nurses also recognized that senior management involvement is a major driving force for the success of quality improvement $(\mathrm{p}<0.001$, Bonferroni $=$ $\mathrm{p}<0.001$ and $\mathrm{p}=0.006$ ).

In terms of activities related to the planning of strategic quality, nurses spent significantly more time on quality improvement programs, compared to para-clinical staff $(\mathrm{p}<0.001)$.

In addition, nurses had more specific goals for their own wards, compared to the other groups. Meanwhile, the administrative employees and managers believed that hospital staff must participate in designing quality improvement programs and that middle managers played a key role in determining quality improvement priorities. The Bonferroni test demonstrated that mean scores quality management activities for managers and administrative employees significantly lower compared to the nurses and Para-clinical staff $(\mathrm{P}<0.001$ and $\mathrm{p}=0.005$ ).

Table 3 Hospital Employees' Attitudes Towards the benefits of accreditation

\begin{tabular}{|l|l|l|l|l|}
\hline Items & Managers/staff & Nurses & Para-clinical staff & P-value \\
\hline Enhanced patient care & $3.14(0.87)$ & $3.68(0.76)$ & $3.05(0.79)$ & $<0.001$ \\
Increase motivation of staff and encourages teamwork and collaboration & $3.15(0.73)$ & $3.58(0.69)$ & $3.08(0.48)$ & $<0.001$ \\
Develop of values shared by all professionals & $3.21(0.83)$ & $3.59(0.91)$ & $3.06(0.81)$ & $<0.001$ \\
Efficient use from internal resources & $3.08(1.19)$ & $3.51(0.77)$ & $3.19(0.88)$ & $<0.001$ \\
Better respond to the populations needs & $3.22(0.84)$ & $3.65(0.83)$ & $3.19(0.71)$ & $<0.001$ \\
Better respond to its partners & $3.16(0.75)$ & $3.48(0.73)$ & $3.02(0.95)$ & $<0.001$ \\
Contribute to the development of collaboration with partners & $3.12(0.84)$ & $3.45(0.83)$ & $3.29(0.71)$ & $<0.001$ \\
Contribute to implementing changes & $3.22(0.97)$ & $3.68(0.68)$ & $3.14(0.70)$ & $<0.001$ \\
\hline
\end{tabular}


Table 4 Quality Improvement-Related Activities in the Iranian Hospital

\begin{tabular}{|l|l|l|l|l|}
\hline Quality Activities & Managers/Staff & Managers/Staff & Para-Clinical Staff & P-value \\
\cline { 2 - 5 } & Mean (SD) & Mean (SD) & Mean (SD) \\
\hline Senior managers commitment and support activities & $3.04(0.69)$ & $3.21(0.68)$ & $2.85(0.77)$ \\
Strategic quality planning activities & $3.23(0.55)$ & $3.32(0.67)$ & $3.15(0.77)$ & $<0.001$ \\
Education and training activities & $2.69(0.87)$ & $2.97(0.82)$ & $2.86(0.86)$ & $<0.001$ \\
Rewards and recognition activities & $3.04(0.88)$ & $3.24(0.82)$ & $3.16(0.74)$ & 0.002 \\
Quality management activities & $2.98(0.64)$ & $3.17(0.67)$ & $3.16(0.68)$ & $<0.001$ \\
Use of data activities & $3.22(0.69)$ & $3.22(0.65)$ & $2.98(0.70)$ & 0.001 \\
Involvement of professionals in accreditation & $3.15(0.83)$ & $3.27(0.68)$ & $3.02(0.67)$ & $<0.001$ \\
\hline
\end{tabular}

According to the results, the mean education score of employees for the implementation of the quality improvement program was below three (of five) in all three groups. Meanwhile, nurses had a significant belief in the area of quality improvement methods, enhancement of performance and occupational skills, and recognized opportunities to improve education quality. Similarly, nurses obtained a higher score in the area of recognition and motivation of staff, compared to the other groups. The Bonferroni test shows that there is a significant difference between the nurses and managers $(\mathrm{p}=0.002)$.

In addition, the mean score of quality management activities was significantly higher in nurses $(p<0.05)$. In terms of information, nurses, as well as administrative employees and managers, had a more positive attitude towards the use of information by hospitals to improve service quality $(p<0.001$; Bonferroni $=p<0.001$ for All). Finally, the attitude of nurses showed that they took part more in accreditation activities compared to the other groups $(\mathrm{p}<0.001$; Bonferroni $=\mathrm{p}<0.001$ and $\mathrm{p}=0.04)$.

\section{Discussion}

This was the first and most comprehensive research performed to evaluate the attitude of hospital employees toward accreditation and quality improvement activities in Iran. Generally, this study showed there was no area in which any of the groups strongly agreed for hospital accreditation in Iran. Moreover, nurses' attitudes towards the impact of accreditation on quality results and the accreditation benefits were more positive compared with the two other groups. In Iran, nurses have the highest input in implementing accreditation standards, compared to other groups (e.g., physicians, staff of the para-clinical wards and administrative employees).

According to the results of the study, nurses had a significantly higher level of participation in implementing accreditation standards, compared to the other groups. Since the accreditation standards are mostly carried out for patient care and safety, this group of hospital employees is mainly responsible for implementing these standards. These observations are also in line with a study conducted by Saadati et $\mathrm{al}^{20}$ who recognized nurse roles, poor cooperation among physicians and administrative staff were some of the issues that nurses experienced during the accreditation process.

Contrary to our findings, Lebanese nurses' perception of quality improvement as a result of accreditation was higher. ${ }^{8}$ It is possible that the low scores are due to the size of the institutions involved in these two different surveys. Our study only included larger hospitals with above 250 beds. Evidence shows that smaller organizations often have a more homogeneous culture and its staff probably shares the same values. ${ }^{23}$ Large-sized hospitals tend to be more hierarchically and bureaucratically organized which makes the implementation of quality work more challenging. ${ }^{8}$

The respondents agree that accreditation is an important tool to improve organizational quality, enhance patient care, increase motivation of staff, encourages teamwork and collaboration, development of values shared by all professionals, efficient use from internal resources, better respond to the populations' needs, better respond to its partners, contribute to the development of collaboration with partners, and contribute to implementing changes. This finding is in line with the results of previous studies. Ehlers et al indicated that accreditation can be an important tool to improve organizational quality. ${ }^{6}$ A study conducted in Brazil, organizational impacts of accreditation were identified through the internal processes, learning and customers. ${ }^{12}$

In addition, the accreditation processes and structure can be another reason for differences in attitudes. Participation in the accreditation process in Iran is mandatory for all hospitals and surveys are performed by the MOHME. Lebanon is 
among the countries in the Eastern Mediterranean region whose accreditation standards have been approved by the International Society for Quality in Health Care and are monitored by international organizations. ${ }^{8}$

The most important barriers and mechanisms for insufficient effectiveness of accreditation were lack of education, insufficient management, and insufficient prioritization of quality work at the hospitals. This is a strong indication that something is not working right and there may be important reasons why respondents think that accreditation only has a low impact on quality results.

Our results also indicate that a lack of education and training are important obstacles to successful implementation. Education and training of staff were critical for the implementation of accreditation. ${ }^{7,8}$ Respondents also saw management as a barrier to successful implementation. In some previous research, leadership and management are highlighted as the most important factor for implementation and effectiveness. ${ }^{6-8,20}$

Our study shows that using a survey method for collecting information on employees' attitudes can be a valuable instrument for "taking the temperature" of an accreditation system. Attitudes seem to reflect aspects of organizational culture and can be both persistent and contagious. $^{2,27}$ Attitudes may be affected by political and public agendas and a biased measure of the impact of accreditation. However, asking employees about their opinions and perceptions can highlight existing problems that managers and decision-makers can then address.

Other recent Iranian studies on the effectiveness of hospital accreditation points in the same direction - only a low/moderate effect on the current system. ${ }^{7,8,12}$ The Iranian hospital accreditation is in its infancy. The responsibility of hospital accreditation in Iran is laid in the hands of OMAHI at the MOHME. In other words, a governmental institute governs the hospital accreditation program in the country. About $70 \%$ of Iranian hospitals are publicly owned and run by the government. ${ }^{28}$ Thus, if the accreditation body's structure and governance is not independent, there is a chance of having biased results. Other countries have a much longer experience of accreditation programs and in Iran, it may take time to implement complex organizational and managerial processes of accreditation. ${ }^{18}$ This is why follow-up studies on attitudes could be used to support the implementation process, e.g. every second or third year. The accreditation assessment methodology is inadequate in Iran because surveys are carried out periodically and over a short period of time. ${ }^{29}$
Respondents did see management as a barrier to successful implementation. Previous studies identified management as the single most important factor for implementation and effectiveness. $^{6-8,18,20,30}$ Senior hospital managers should be made aware of the benefits of accreditation to guarantee their commitment and leadership in order to make quality transformations to services. They should provide necessary resources for improving the quality, safety and effectiveness of hospital services. According to Donabedian, any system for quality improvement could potentially be used successfully to improve quality but could also fail without proper management - the systems' effectiveness depends on management commitment and how the methods for accreditation are implemented and governed during daily clinical practice. ${ }^{31}$

\section{Strengths and Limitations}

Although the current study had several strengths (e.g. multisite, large sample size and the high response rates across the hospitals) there are several limitations that need to be addressed. Firstly, our study is cross-sectional, and is an attitude survey, with the attendant limitations of such studies, including potential for self-selection and bias. Secondly, since this study explored the attitudes of staff towards the accreditation process, conclusions had to be drawn. This could be a limitation since the study, to some extent, examined staff's personal perceptions and attitudes. A third limitation to our study is, of course, that physicians did not participate in the survey. The physicians are the most influential and powerful group in Iran's hospital healthcare system, as is the case almost globally. ${ }^{32}$ A specific justification for this situation could be the fact that they have the most senior positions in the overall governance structure of the health sector in the country (i.e. in the MOHME). Fourthly, we only selected teaching hospitals. Therefore, the findings do not represent all hospitals in Iran and more research in this area is needed before we are able to generalize our findings. Finally, the missing data resulted from the un-returned questionnaires is a potential limitation. People, who do not respond, may have different attitudes compared to those who do respond. We could not, however, look further into this problem in our study. We have not collected any information on non-responders.

\section{Conclusions}

Our results show that studying attitudes may be important for understanding the effectiveness of accreditation. The study showed there was no area in which any of the groups strongly agreed for hospital accreditation in Iran. Also, nurses' attitudes towards the accreditation benefits were more positive 
compared with the two other groups. In this current study, the most important barriers and mechanisms for insufficient effectiveness of accreditation were lack of education, insufficient management, and insufficient prioritization of quality work at the hospitals. Therefore, managers and policymakers should consider education, sufficient management, and prioritization of quality activities as major components for the successful implementation of accreditation. According to the results, more attention needs to be paid to employees' attitudes towards accreditation. These attitudes will reveal the realities of healthcare systems and can influence government decisions. We can recommend that the questionnaire is used again in Iran in a few years' time to see if attitudes have changed. Finally, assessing the impact of accreditation on patient safety and quality care indicators could also be considered in future studies.

\section{Ethics Approval and Consent to Participate}

The study protocol was approved by the central ethical committees of Iran university of Medical Sciences (IR.IUMS.REC 1395.25599). Before completing the questionnaire, all participants provided written consent to participate in the study.

\section{Acknowledgments}

The authors would like to thank all the participants in the survey for their time and effort. We also thank Michele Brailsford for assistance with language editing and proofreading that greatly improved the manuscript.

\section{Funding}

The research was funded by Iran University of Medical Science.

\section{Disclosure}

The authors declare that they have no competing interests.

\section{References}

1. Wardhani V, van Dijk JP, Utarini A. Hospitals accreditation status in Indonesia: associated with hospital characteristics, market competition intensity, and hospital performance? BMC Health Serv Res. 2019;19 (1):372. doi:10.1186/s12913-019-4187-x

2. Andres E, Song W, Song W, Johnston J. Can hospital accreditation enhance patient experience? Longitudinal evidence from a Hong Kong hospital patient experience survey. BMC Health Serv Res. 2019;19:623. doi:10.1186/s12913-019-4452-z

3. Brubakk K, Vist GE, Bukholm G, Barach P, Tjomsland O. A systematic review of hospital accreditation: the challenges of measuring complex intervention effects. BMC h Health Serv Res. 2015;15 (1):280. doi:10.1186/s12913-015-0933-x
4. Greenfield D, Pawse M, Braithwaite J. The role and impact of accreditation on the healthcare revolution. Revista Acreditação. 2012;1(2):134-150.

5. Bahadori M, Teymourzadeh E, Ravangard R, Saadati M. Accreditation effects on health service quality: nurse viewpoints. Int $J$ Health Care Qual Assur. 2018;31(7):697-703. doi:10.1108/IJHCQA-07-2017-0126

6. Ehlers LH, Jensen MB, Simonsen KB, Rasmussen GS, Braithwaite J. Attitudes towards accreditation among hospital employees in Denmark: a cross-sectional survey. Int J Qual Health Care. 2017;29 (5):693-698. doi:10.1093/intqhe/mzx090

7. El-Jardali F, Hemadeh R, Jaafar M, et al. The impact of accreditation of primary healthcare centers: successes, challenges and policy implications as perceived by healthcare providers and directors in Lebanon. BMC Health Serv Res. 2014;14(1):86. doi:10.1186/1472-6963-14-86

8. El-Jardali F, Jamal D, Dimassi H, Ammar W, Tchaghchaghian V. The impact of hospital accreditation on quality of care: perception of Lebanese nurses. Int J Qual Health Care. 2008;20(5):363-371. doi:10.1093/intqhe/mzn023

9. Greenfield D, Pawsey M, Naylor J, Braithwaite J. Are accreditation surveys reliable? Int J Health Care Qual Assur. 2009;22(2):105-116. doi:10.1108/09526860910944601

10. Nekoei-Moghadam M, Amiresmaili M, Iranemansh M, Iranmanesh M. Hospital accreditation in Iran: a qualitative case study of Kerman hospitals. Int J Health Plann Manag. 2018;33 (2):426-433. doi:10.1002/hpm.2480

11. Sack C, Scherag A, Lütkes P, Günther W, Jöckel K-H, Holtmann G. Is there an association between hospital accreditation and patient satisfaction with hospital care? A survey of 37000 patients treated by 73 hospitals. Int J Qual Health Care. 2011;23(3):278-283. doi:10.1093/intqhe/mzr011

12. Saut AM, Berssaneti FT, Moreno MC. Evaluating the impact of accreditation on Brazilian healthcare organizations: a quantitative study. Int J Qual Health Care. 2017;29(5):713-721. doi:10.1093/ intqhe/mzx094

13. Lam MB, Figueroa JF, Feyman Y, Reimold KE, Orav EJ, Jha AK. Association between patient outcomes and accreditation in US hospitals: observational study. $B M J$. 2018;363:k4011. doi:10.1136/bmj. k4011

14. Yildiz A, Kaya S. Perceptions of nurses on the impact of accreditation on quality of care: a survey in a hospital in Turkey. Clin Gov Int J. 2014;19(2):69-82. doi:10.1108/CGIJ07-2013-0021

15. Reisi N, Raeissi P, Sokhanvar M, Kakemam E. The impact of accreditation on nurses' perceptions of quality of care in Iran and its barriers and facilitators. Int $J$ Health Plann Manag. 2019;34(1):230-240. doi:10.1002/hpm.2642

16. Arab-Zozani M, Sokhanvar M, Kakemam E, Didehban T, Hassanipour S. History of health technology assessment in Iran. Int J Technol Assess Health Care. 2020;36(1):34-39. doi:10.1017/ S0266462319003489

17. Raeissi P, Sokhanvar M, Kakemam E. Outsourcing in Iranian hospitals: findings from a qualitative study. Int $J$ Health Plann Manag. 2018;33(4):e1250-e1261. doi:10.1002/hpm.2619

18. Yousefinezhadi T, Mosadeghrad AM, Mohammad A, Ramezani M, Sari AA. An analysis of hospital accreditation policy in Iran. Iran J Public Health. 2017;46(10):1347-1358.

19. Aryankhesal A, Sheldon T. Effect of the Iranian hospital grading system on patients' and general practitioners' behaviour: an examination of awareness, belief and choice. Health Serv Manag Res. 2010;23(3):139-144. doi:10.1258/hsmr.2009.009028

20. Saadati M, Bahadori M, Teymourzadeh E, Ravangard R, Alimohammadzadeh K, Mojtaba Hosseini S. Accreditation in one teaching hospital: a phenomenology study among Iranian nurses. Int J Health Care Qual Assur. 2018;31(7):855-863. doi:10.1108/ IJHCQA-08-2017-0150 
21. Hashjin AA, Kringos DS, Manoochehri J, Aryankhesal A, Klazinga NS. Development and impact of the Iranian hospital performance measurement program. BMC Health Serv Res. 2014;14 (1):448. doi:10.1186/1472-6963-14-448

22. Pomey M-P, Contandriopoulos A-P, François P, Bertrand D. Accreditation: a tool for organizational change in hospitals? Int J Health Care Qual Assur. 2004;17(3):113-124. doi:10.1108/ 09526860410532757

23. Shortell SM, O'Brien JL, Carman JM, et al. Assessing the impact of continuous quality improvement/total quality management: concept versus implementation. Health Serv Res. 1995;30 (2):377.

24. Polit DF, Beck CT, Owen SV. Is the CVI an acceptable indicator of content validity? Appraisal and recommendations. Res Nurs Health. 2007;30(4):459-467. doi:10.1002/nur.20199

25. DeVon HA, Block ME, Moyle-Wright P, et al. A psychometric toolbox for testing validity and reliability. J Nurs Scholarsh. 2007;39 (2):155-164. doi:10.1111/j.1547-5069.2007.00161.x

26. DeVellis RF. Scale Development: Theory and Applications. California: Sage publications; 2016.

27. Braithwaite J, Herkes J, Ludlow K, Testa L, Lamprell G. Association between organisational and workplace cultures, and patient outcomes: systematic review. BMJ Open. 2017;7(11):e017708. doi:10.1136/bmjopen-2017-017708
28. Kakemam E, Goodall AH. Hospital performance and clinical leadership: new evidence from Iran. BMJ Leader. 2019;1-7. doi:10.1136/ leader-2019-000160

29. Teymourzadeh E, Ramezani M, Arab M, Foroushani AR, Sari AA. Surveyor management of hospital accreditation program: a thematic analysis conducted in Iran. Iran Red Crescent Med J. 2016;18(5): e30309. doi:10.5812/ircmj.30309

30. Almasabi M, Thomas S. The impact of Saudi hospital accreditation on quality of care: a mixed methods study. Int J Health Plann Manag. 2017;32(4):e261-e278. doi:10.1002/hpm.2373

31. Donabedian A. The effectiveness of quality assurance. Int J Qual Health Care. 1996;8(4):401-407. doi:10.1093/intqhe/8.4.401

32. Flood AB, Fennell ML. Through the lenses of organizational sociology: the role of organizational theory and research in conceptualizing and examining our health care system. J Health Soc Behav. 1995;35:154-169. doi:10.2307/2626962
Journal of Multidisciplinary Healthcare

\section{Publish your work in this journal}

The Journal of Multidisciplinary Healthcare is an international, peerreviewed open-access journal that aims to represent and publish research in healthcare areas delivered by practitioners of different disciplines. This includes studies and reviews conducted by multidisciplinary teams as well as research which evaluates the results or conduct of such teams or healthcare processes in general. The journal

\section{Dovepress}

covers a very wide range of areas and welcomes submissions from practitioners at all levels, from all over the world. The manuscript management system is completely online and includes a very quick and fair peer-review system. Visit http://www.dovepress.com/testimonials. php to read real quotes from published authors. 